\title{
zonafunces
}

\section{DAICH, Deborah, VARELA, Cecilia (Coordinadoras) (2020) Los feminismos en la encrucijada del punitivismo. Buenos Aires, Biblos, 238 pp.}

Los feminismos en la encrucijada del punitivismo, coordinado por Deborah Daich y Cecilia Varela, es un aporte de investigaciones y reflexiones que echan luz sobre las demandas y respuestas en torno a las violencias de género y sexuales.

La problematización y visibilización de las violencias en la última década ha llevado a un auge de demandas y respuestas de carácter punitivo. En este contexto, las coordinadoras del libro se plantean, "preguntas en torno a la relación que se tejía entre feminismos, instrumentos penales y justicia: ¿es el ámbito penal y su esquema de víctimas y victimarios una forma adecuada para pensar y resolver conflictos?, ¿cómo producir leyes que protejan a las mujeres sin decantar en inflación penal?, (...) ¿lo penal, es una herramienta, una forma de imaginar un conflicto, o ambas? (...). Éstas y otras inquietudes, son exploradas en este libro desde perspectivas asociadas a (trans)feminismos críticos, anti-punitivos, interseccionales, e informados por investigaciones etnográficas.

El primer capítulo, a cargo Tamar Pitch, titulado "Feminismo punitivo" abre el libro con una serie de problematizaciones que serán retomadas por las demás autoras de la compilación. Pitch define al "feminismo punitivo" como "las movilizaciones que, apelando al feminismo y la defensa de las mujeres, se vuelven protagonistas de pedidos de criminalización", y a partir de su análisis de las demandas por la prohibición de la gestación subrogada y de las demandas que exigen la penalización del cliente de la prostitución, va a resaltar las afinidades de este giro punitivo de cierto feminismo con la racionalidad neoliberal. Señala que la centralidad del lenguaje de la violencia y la victimización en las demandas contemporáneas del feminismo ha suplantando un lenguaje propio de un feminismo de la segunda ola en donde "explotación", "opresión", "dominación" eran centrales a un paradigma revolucionario, “(...) arriesgándose a contribuir a la reducción de la

Revista Zona Franca- Centro de estudios interdisciplinario sobre las mujeres (CEIM)- Maestría poder y sociedad desde la problemática de género (MG), Rosario, Argentina. ISSN, 2545-6504 http://zonafranca.unr.edu.ar/index.php/ZonaFranca| Número 29 (2021). 
política, justamente, a la política penal". En este proceso, es el "feminismo institucional" el que gana reconocimiento e inserción en los aparatos estatales, hablando en nombre de las mujeres que identifica como víctimas. Sin negar las violencias sufridas por las mujeres y todo lo que el feminismo ha logrado en búsqueda de justicia, Pitch finaliza su texto con dos reflexiones, por un lado, muestra cómo las demandas punitivas impulsadas por ciertos feminismos reproducen los vicios e ilusiones de una política tradicional que confía en la justicia penal como solución a todos los males y una oportunidad para políticas carcelarias. Por el otro, señala que aún si consideramos la prostitución y la gestación subrogadas como "dañinas" no resulta de ello necesariamente la prohibición penal como medio único o privilegiado para combatirlas.

El segundo capítulo, "Feminismo y derecho penal, una relación penosa", escrito por Dolores Juliano, también aborda la conflictiva relación de los feminismos con el derecho penal. Juliano se apoya en una trayectoria de investigación con mujeres de grupos subalternos y "desviados" para dialogar críticamente con las aspiraciones contemporáneas del feminismo. Para esta autora el carácter androcéntrico del derecho penal torna peligrosas las crecientes demandas de algunos sectores del feminismo apelando al derecho penal para proteger los derechos de las mujeres. Señala que las políticas represivas no resuelven las causas de los problemas, más bien, sirven para extremar el control sobre los sectores menos privilegiados de la sociedad, entre ellos, las mujeres que no se adecúan a los estereotipos de género y de buenas víctimas. Aquí Juliano reflexiona sobre la conflictiva relación que han tenido los feminismos con las mujeres "infractoras" y pertenecientes a sectores subalternos y estigmatizados (presas, trabajadoras sexuales, etc.). Pone en duda la vocación paternalista de ciertos feminismos que apelan al derecho penal para proteger a estos sectores; y reivindica la apuesta política de reconocer a las mujeres de sectores subalternos como interlocutoras válidas capaces de articular demandas propias.

Revista Zona Franca- Centro de estudios interdisciplinario sobre las mujeres (CEIM)- Maestría poder y sociedad desde la problemática de género (MG), Rosario, Argentina. ISSN, 2545-6504 http://zonafranca.unr.edu.ar/index.php/ZonaFranca| Número 29 (2021). 
El siguiente texto, "El discurso hegemónico sobre el acoso sexual" de Marta Lamas ofrece una reflexión crítica sobre el discurso hegemónico en torno al acoso sexual, articulado con fuerza a partir de la eclosión del \#MeToo en Estados Unidos e irradiado al resto del globo. Lamas nos recuerda que todo discurso tiene una historia, siendo éste una elaboración específicamente norteamericana, donde las teorizaciones de una vertiente del feminismo, el feminismo de la dominación, formado a mediados de los 70 interactuó con la tradición legal estadounidense y con un persistente puritanismo. Lamas nos pone en alerta ante la afinidad entre punitivismo y puritanismo sexual, y cómo se articulan en una constante producción de las mujeres como víctimas y la sexualidad como algo intrínsicamente peligroso. Este paradigma para la autora corre el riesgo de "sanitizar" el espacio de trabajo, promover pánicos sexuales y facilitar el disciplinamiento de la fuerza de trabajo. Llama a reconocer la extensión de las prácticas de acoso sexual, pero también a reconocer que hay más de una manera de elaborar un discurso en torno a ello, así como de respuestas posibles, más allá de la punitiva.

El cuarto aporte del libro, escrito por Virginia Cano, se titula "Afecciones punitivas e imaginación política: des-bordes de la lengua penal' y recupera los análisis críticos de las tendencias criminalizadoras junto con los estudios sobre economías afectivas para arrojar una mirada reflexiva sobre los procesos de problematización de las violencias sexuales y de género dentro de los movimientos feministas y LGTBI. Cano retoma la propuesta de Tamar Pitch que llama a analizar la relación entre la construcción de un problema social y su criminalización teniendo en cuenta que el problema es construido en vistas al tipo de solución que parece disponible o que se tiene en mente. Así la creciente judicialización de las relaciones sociales y la expansión del derecho penal crea una matriz de pensamiento y acción que desborda el ámbito estrictamente jurídico de lo penal para "seguir hegemonizando nuestra imaginación política, burocratizando nuestros modos de intervenir en los complejos entramados de violencia y vulnerabilidad, y modelando nuestros cuerpos y sus economías afectivas." La lógica binaria del derecho penal

Revista Zona Franca- Centro de estudios interdisciplinario sobre las mujeres (CEIM)- Maestría poder y sociedad desde la problemática de género (MG), Rosario, Argentina. ISSN, 2545-6504 http://zonafranca.unr.edu.ar/index.php/ZonaFranca| Número 29 (2021). 
que enfatiza la responsabilidad individual en los conflictos y la construcción de víctimas y victimarios como posiciones exclusivas y excluyentes, simplifica la complejidad de las relaciones sociales atravesadas por la violencia, al mismo tiempo que oculta las responsabilidades comunitarias en donde sucede. Cano aborda dos prácticas y discursos dentro de organizaciones feministas y de disidencia sexual: los escraches y denuncias públicas por violencia sexual y/o de género y las estrategias de expulsión en espacios activistas ante denuncias de violación o abuso. Reconociendo el valor que para muchas personas tienen dentro de los colectivos como estrategias de denuncia y reparación, sostiene la necesidad de revisar críticamente estas prácticas, atendiendo a sus límites y miopías. Señalará que alientan la individualización de las responsabilidades borrando las responsabilidades colectivas, la alterización de la violencia como algo como algo ajeno y un deseo de seguridad afín a las formas de gobierno neoliberal que busca segregar y controlar individuos y poblaciones. Cano indicará la necesidad de estrategias de intervención ante las violencias que hagan énfasis en las responsabilidades comunes (aunque diferenciales) con una perspectiva sensible a la vulnerabilidad e interdependencia compartida.

El quinto capítulo, "Los feminismos entre la política de cifras y la experticia en violencia de género" con la autoría de Catalina Trebisacce y Cecilia Varela aborda un caso de construcción de saber sobre la violencia de género, articulado alrededor de las cifras de femicidios contabilizadas por una organización de la sociedad civil. El caso analizado por las autoras, el de un observatorio que se dedica a contabilizar casos de femicidios en Argentina, se caracteriza por una cuantificación que carece de rigor metodológico y cuyo principal objetivo es el de visibilizar una problemática al mismo tiempo que posiciona a la organización dentro de un campo de experticia. A partir de este caso se preguntan sobre los límites de visibilizar y conceptualizar la violencia de género en términos de una política de cifras y de mera cuantificación de casos. Señalan que esta lógica de los observatorios orientada a la producción de cifras, homogeniza las violencias contra las mujeres, descontextualizan los

Revista Zona Franca- Centro de estudios interdisciplinario sobre las mujeres (CEIM)- Maestría poder y sociedad desde la problemática de género (MG), Rosario, Argentina. ISSN, 2545-6504 http://zonafranca.unr.edu.ar/index.php/ZonaFranca| Número 29 (2021). 
casos, los simplifican como eventos discretos organizados en torno a la diada víctima-victimario y reducen la conflictividad a la mera opresión de género por fuera de las dinámicas interseccionales. Reivindican la construcción de un conocimiento guiado por las epistemologías feministas, que frente a las estrategias de observarcuantificar-visibilizar, priorice saberes situados y plurales, capaces de escuchar y narrar experiencias de mujeres y poblaciones diversas y subalternas.

Por su parte, Agustina Iglesias Skulj en su texto "Performance de la fragilidad $y$ el empoderamiento: reflexiones en torno del feminismo punitivo" se propone cultivar la crítica de la instrumentalización del sistema penal por parte del feminismo institucional desde una criminología (trans)feminista. Skulj señala la necesidad de construir "estrategias de distanciamiento" desde una criminología transfeminista que permitan ampliar y complejizar el campo de visión que aportó la categoría género dentro de los feminismos. En este sentido apela al método interseccional, para cuestionar las categorías sobre las que se define la violencia de género en base a una experiencia universalizante y esencialista del "hecho de ser mujer". Para la autora es crítico preguntarse entonces por los "criterios de selectividad respecto de qué experiencias pueden ser politizadas" y sobre las que se producen saberes y legitiman intervenciones institucionales contra la violencia de género, y por los efectos materiales de estas definiciones esencialistas y universalizantes de la violencia y el género.

La contribución subsiguiente, "Qué hubo de nuevo en la legislación penal argentina contra la trata de personas? Un primer análisis de lo que nos dejaron las leyes 26.364 y 26.842." con firma de Marisa Tarantino es un balance crítico de la legislación penal anti-trata, la discusión doctrinaria en torno a los tipos penales y jurisprudencia asociada. Más allá de la valiosa disección de los tipos penales introducidos por las leyes anti-trata, Tarantino explora cómo en este campo jurídico las categorías de "vulnerabilidad", "consentimiento" y "dignidad" fueron perfiladas por el sistema de justicia bajo un paradigma neo-abolicionista de la prostitución que ha sido presentando como la única perspectiva de género y feminista en torno al

Revista Zona Franca- Centro de estudios interdisciplinario sobre las mujeres (CEIM)- Maestría poder y sociedad desde la problemática de género (MG), Rosario, Argentina. ISSN, 2545-6504 http://zonafranca.unr.edu.ar/index.php/ZonaFranca| Número 29 (2021). 
comercio sexual. Siguiendo a la autora, este ocultamiento de diversas perspectivas en torno a la prostitución/trabajo sexual no sólo invisibiliza otros feminismos y perspectivas, también oculta y silencia la voz de las trabajadoras sexuales y sus reclamos políticos.

Dentro de la misma problemática en torno a la trata de personas, los dos últimos capítulos traen material empírico sobre mujeres que han sido objeto de las intervenciones impulsadas por las políticas anti-trata.

En "¿Todas las mujeres acusadas de tráfico internacional de drogas son víctimas de trata de personas? Género, clase, raza y nacionalidad en los discursos sobre crimen organizado en Brasil", Natalia Corazza Padovani analiza la producción discursiva de categoría de "víctimas", "criminales" y "crimen organizado" en su intersección de nociones de género, raza, clase y nacionalidad. Analizando los discursos humanitarios y de seguridad, y luego apoyándose en un estudio de caso, Padovani muestra cómo la categorización de aquellas mujeres acusadas de ser mulas en el tráfico de drogas como víctimas de trata de personas es afín a un feminismo carcelario, que en el afán de ayudar a "mujeres vulnerables" ignora las relaciones sociales y afectivas de éstas, así como sus capacidades para actuar dentro de mercados criminalizados. El artículo de Padovani nos recuerda que las buenas intenciones que promueven identificar a mujeres cómo víctimas en el marco de su participación en actividades criminalizadas no son suficientes, e incluso son contraproducentes, si no somos capaces de desmontar las categorías que en nudos interseccionales producen "víctimas" y "criminales."

El libro cierra con "De mujeres de la noche y madamas a proxenetas. Una crítica feminista al punitivismo antitrata", de Melisa Cabrapan Duarte. En este trabajo de naturaleza etnográfica, la autora reconstruye la trayectoria de vida y laboral de mujeres que viviendo en una localidad petrolera de la Patagonia ejercieron el trabajo sexual en locales comerciales para luego pasar a ser madamas en estos mismos sitios. Según los relatos y experiencias personales de las entrevistadas en torno a

Revista Zona Franca- Centro de estudios interdisciplinario sobre las mujeres (CEIM)- Maestría poder y sociedad desde la problemática de género (MG), Rosario, Argentina. ISSN, 2545-6504 http://zonafranca.unr.edu.ar/index.php/ZonaFranca| Número 29 (2021). 
sus trayectorias laborales, las prácticas y movilidades dentro de condicionamientos genéricos y económicos, las llevaron a insertarse primero como mujeres de la noche para luego pasar al rol de madamas, con el paso del tiempo que lleva a un decrecimiento del capital erótico, pero también un incremento del capital económico y de experiencia en la gestión de establecimientos. El conjunto de experiencias y significados construidos por las mujeres en sus contextos específicos, le permite a Cabrapan Duarte desafiar las lógicas penales de "victimas" y "criminales" y problematizar la matriz punitiva de las políticas anti-trata que buscan penalizar cualquier organización comercial del sexo, y que, en el mismo proceso, criminalizan a las mismas mujeres que intentan rescatar.

Los feminismos en la encrucijada del punitivismo, frente a las emergencias y urgencias declaradas ante las violencias que suelen decantar por las respuestas punitivas, es una compilación oportuna que aporta reflexiones, conceptos y experiencias para cuestionar las lógicas criminalizadoras y comenzar a delinear respuestas alternativas. De metodologías, enfoques y disciplinas diversas, el libro coordinado por Daich y Varela, logra mantener una coherencia y diálogo interno entre los diversos capítulos que hace aún más provechosa su lectura.

Felipe González*

*Licenciado en Ciencia Política por la Universidad de Buenos Aires. Contacto: felipefg1@hotmail.com

Revista Zona Franca- Centro de estudios interdisciplinario sobre las mujeres (CEIM)- Maestría poder y sociedad desde la problemática de género (MG), Rosario, Argentina. ISSN, 2545-6504 http://zonafranca.unr.edu.ar/index.php/ZonaFranca| Número 29 (2021). 